\title{
A Distributed Parameter Model Approximation Method
}

\author{
Pyakillya B.I. \\ Department of Integrated Computer Control Systems, National Research Tomsk Polytechnic \\ University, Tomsk, Russia
}

Keywords: Approximation, transfer function, irrational transfer function, distributed parameter system, interpolation.

\begin{abstract}
At present, many control theory scientists and control engineers work with controller design problem for distributed parameter system because many real industrial systems have distribution of its parameters. The main difficulty is caused by using the mathematical description in terms of irrational transfer functions which doesn't let to use existing design algorithms. It opens the way for approximation techniques. This paper presents a method for distributed parameter model approximation and enables to solve this problem with low computational costs.
\end{abstract}

\section{Introduction}

The approximation problem for distributed parameter systems has a high importance in control theory field. Many scientists and engineers work with this problem and achieve important results [1]. Problem's importance is caused by controller design goals for industrial plants which have distributed parameters. For instance, these parameters may be temperature of a rod or deflection of a beam [1]. These systems are described by partial-differential equations (PDE) and often have second order partial derivatives. The transfer functions of distributed parameter systems (DPS) are irrational functions in contrast to lumped-parameter systems which are described by rational transfer functions. It brings complexity to controller design problems, because the main part of controller design algorithms relates to rational transfer function descriptions. Irrational transfer functions have infinitely many poles and zeros and it's one of the problems which make analysis much more difficult than in rational transfer functions case. This is why researches using the approximation techniques which can help to understand main distributed parameter systems properties and make their analysis simpler. It gives the possibility to develop a controller and get the best performance of control system. This paper presents a distributed parameter model approximation method which is based on Real Interpolation method (RIM) [2,3].

\section{A Real Interpolation Method Application to Approximation Problem}

The RIM is a method which operates with mathematical descriptions in the integral transforms field. The basis of this method is the real integral transform:

$$
F(\delta)=\int_{0}^{\infty} f(t) \cdot e^{-\delta t} d t, \delta \in[C, \infty), C \geq 0,
$$

where the original function $f(t)$ is associated with its integral transform $F(\delta)$, presented as a function of real variable $\delta \in[C, \infty)$, when $C \geq 0$. The integral transform (1) can be considered as a special case of the Laplace transform when the complex variable $s=\delta+j \omega$ is replaced by real variable $\delta, \omega=0$.

Using the transform (1) as in the case of the Laplace transform, we can achieve a goal, that is to obtain plant's transfer function $W(s)$ which is ratio between Laplace transforms of an input signal $x(t)$ and an output signal $y(t)[4]$ : 


$$
W(s)=\frac{b_{m} s^{m}+b_{m-1} s^{m-1}+\ldots+b_{1} s+b}{a_{n} s^{n}+a_{n-1} s^{n-1}+\ldots+a_{1} s+1}, m<n,
$$

where $m$ - is numerator's order, $n$ - is denominator's order.

According to this goal, first of all we have to get a real transfer function description. The transition to real transfer function is performed by formal replacement of a complex variable $s$ by a real variable $\delta$ :

$$
W_{s \rightarrow \delta}(\delta)=\frac{b_{m} \delta^{m}+b_{m-1} \delta^{m-1}+\ldots+b_{1} \delta+b}{a_{n} \delta^{n}+a_{n-1} \delta^{n-1}+\ldots+a_{1} \delta+1}
$$

A following stage in solving the approximation problem is to obtain the unknown coefficients $d_{i}, i=1, . . m_{r}, c_{j}, j=1, . . n_{r}$, of the reduced real transfer function:

$$
W_{r}(\delta)=\frac{d_{m_{r}} \delta^{m_{r}}+d_{m_{r}-1} \delta^{m_{r}-1}+\ldots+d_{1} \delta+d}{c_{n_{r}} \delta^{n_{r}}+c_{n_{r}-1} \delta^{n_{r}-1}+\ldots+c_{1} \delta+1}, n_{r} \leq n, m_{r} \leq m
$$

This can be done using the numerical characteristic $\left\{W\left(\delta_{i}\right)\right\}_{i=1, . . \eta}$ and a system of linear equations. The interpolation nodes $\delta_{i}$ are chosen from range where function (3) has a significant change.

For this purpose we have to write a system of linear algebraic equations:

$$
W\left(\delta_{i}\right)=\frac{d_{m_{r}} \delta_{i}^{m_{r}}+d_{m_{r}-1} \delta_{i}^{m_{r}-1}+\ldots+d_{1} \delta_{i}+d}{c_{n_{r}} \delta_{i}^{n_{r}}+c_{n_{r}-1} \delta_{i}^{n_{r}-1}+\ldots+c_{1} \delta_{i}+1}, i=1, . . \eta,
$$

where $\eta$ is a number of unknown coefficients, $\eta=n_{r}+m_{r}+1$.

In a left part of (5) there are placed the elements of a numerical characteristic $\left\{W\left(\delta_{i}\right)\right\}_{i=1, . . \eta}$.

In a right part of (5) we have the unknown coefficients and known interpolation nodes $\delta_{i}$.

As a result of system of linear algebraic equations solution we get a reduced mathematical model of a plant in terms of a real transfer function $W(\delta)$.. The transition to (2) is performed by another formal replacement of a real variable $\delta$ by a complex variable $s[2,3]$.

The obtained model quality can be checked using following criteria:

$$
\begin{aligned}
& \Delta=\max _{1<i<N}|| W\left(j \omega_{i}\right)|-| W_{m}\left(j \omega_{i}\right) \mid, \\
& \Delta=\sum_{i=1}^{N}|| W\left(j \omega_{i}\right)|-| W_{m}\left(j \omega_{i}\right) \mid, \\
& \Delta=\sum_{i=1}^{N}\left(\left|W\left(j \omega_{i}\right)\right|-\left|W_{m}\left(j \omega_{i}\right)\right|\right)^{2} ;
\end{aligned}
$$

where $W(j \omega)$ - is a Fourier transfer function of a given model, $W_{m}(j \omega)$ - is a Fourier transfer function of an obtained model. Also, these criteria can be used in time domain.

This method can be used for distributed parameter system models approximation. Main difference is in view of a given transfer function. In lumped-parameter system case we have a rational transfer function, in distributed parameter system case we have an irrational transfer function. But ways of obtaining the numerical characteristic and solving the system of linear equations are the same. In next part will be presented the distributed parameter system case.

\section{The Distributed parameter System Case}


Suppose we have a vibrating string of some length $L$ with dissipation. This plant is described by PDE [5]:

$$
\frac{\partial^{2} z(x, t)}{\partial t^{2}}+\varepsilon\left\langle\frac{\partial z(., t)}{\partial t}, b\right\rangle b(x)=\frac{\partial^{2} z(x, t)}{\partial x^{2}}+b(x) u(t),
$$

where $z(x, t)$ - is the deflection at time $t$ and at position $x$ along the string,,$\varepsilon$-damping parameter.

This equation (6) is an expanded version of wave equation which has the form

$$
\frac{\partial^{2} z(x, t)}{\partial t^{2}}=c^{2} \frac{\partial^{2} z(x, t)}{\partial x^{2}}, 0<x<L, t \geq 0 .
$$

The constant $c=\frac{\tau}{\rho}$ where $\tau-$ is she string tension, $\rho-$ is the density. This equation (7) describes many other situations such as acoustic plane waves, lateral vibrations in beams, and electrical transmission lines [6]. If we consider appropriate boundary conditions $z(0, t), z(L, t)$ and initial conditions $z(., 0), \frac{\partial z(., 0)}{\partial t}$ then we will obtain the irrational transfer function of this vibrating string which takes into account damping property [1]:

$$
W(s)=\frac{\frac{s}{2} \cdot \sinh (s)+2 \cosh \left(\frac{s}{2}\right)-3 \cosh ^{2}\left(\frac{s}{2}\right)+1}{s\left(s+\frac{\varepsilon}{2}\right) \sinh (s)+\varepsilon\left(\frac{s}{2} \cdot \sinh (s)+2 \cosh \left(\frac{s}{2}\right)-3 \cosh ^{2}\left(\frac{s}{2}\right)+1\right)},
$$

where $s-$ is the complex variable.

Then we have to get the the numerical characteristic of (8). Firstly, we obtain the real transfer function representation of (8):

$$
W(\delta)=\frac{\frac{\delta}{2} \cdot \sinh (\delta)+2 \cosh \left(\frac{\delta}{2}\right)-3 \cosh ^{2}\left(\frac{\delta}{2}\right)+1}{\delta\left(\delta+\frac{\varepsilon}{2}\right) \sinh (\delta)+\varepsilon\left(\frac{\delta}{2} \cdot \sinh (\delta)+2 \cosh \left(\frac{\delta}{2}\right)-3 \cosh ^{2}\left(\frac{\delta}{2}\right)+1\right)} .
$$

Next step is solving the system of linear equation (5). In result we obtain the best description in terms of rational transfer function:

$$
W_{m}(s)=\frac{0,059 s^{2}-0,143 s+0,043}{0,119 s^{3}+0,13 s^{2}+0,152 s+1} .
$$

The approximation criterion is a maximum absolute difference between frequency responses of the given irrational transfer function and the obtained rational transfer function:

$$
\Delta=\max || W(j \omega)|-| W_{m}(j \omega)|| \cdot 100=37,6 .
$$

This value tells that approximation result is satisfactory and the obtained model can be used for simple controller design objectives. A better result can be achieved by using of an iterative approximation procedure with interpolation nodes change.

In the Fig. 1 are displayed the plots of frequency responses for given and obtained model.

\section{Conclusions}

In the proposed paper, the distributed parameter model approximation method is applied to approximation of the vibrating string transfer function. The method is based on Real interpolation 
method and used integral transforms. It enables a way of distributed parameter model approximation and reduces the computational cost using a system of linear algebraic equations. These advantages give the possibility to use this method in first step of controller design algorithms for distributed parameter systems.

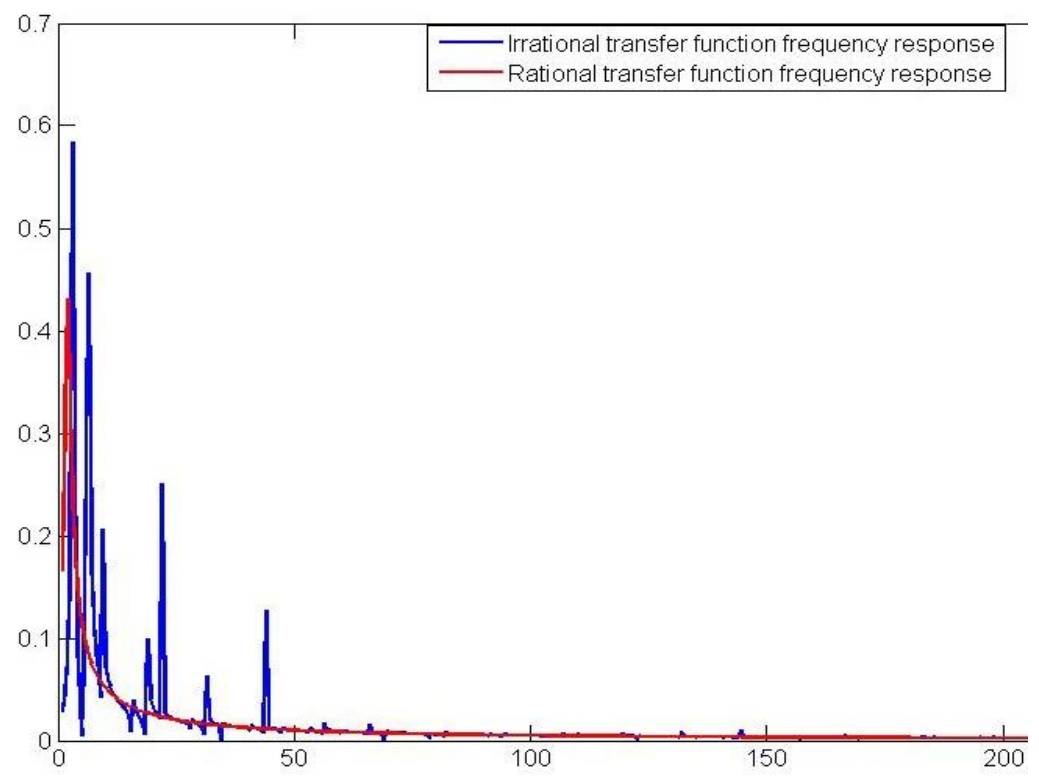

Fig. 1. Frequency responses of transfer functions.

\section{References}

[1] Ruth Curtain, Kirsten Morris. Transfer functions of distributed parameter systems: A tutorial. Automatica, 45, pp. 1101-1116, 2009.

[2] Belikmaier, M.Y, Goncharov V.I. Correctors for automatic control systems: Synthesis by uniform approximation // Automation and Remote control, 5, pp. 715-721, 1997.

[3] Goncharov V. Rudnicki V. Real interpolation method in automatic control systems selfadjustment problem// Systems Science, 3(36), pp. 35 - 37, 2010.

[4] Dorf R., Bishop R. Modern control systems. Prentice Hall, 2011.

[5] Guenther, R. B., \& Lee, J. W. Partial differential equations of mathematical physics and integral equations. Edgewood Cliffs, NJ: Prentice-Hall, 1988.

[6] Towne, D. H. Wave phenomena. New York: Dover Publications, 1988. 